\title{
Post-buckling shapes of a Blood Vessel Embedded in Nonlinear Soft Tissue
}

\author{
${ }^{1}$ Mohammed Elgindi, ${ }^{2}$ Dongming Wei, $*{ }^{1,3}$ Mohammed Ghazy \\ ${ }^{1}$ Science Department, Texas A\&M University, Qatar Campus, Doha 23874, Qatar \\ ${ }^{2}$ Department of Mathematics, University of New Orleans, New Orleans, LA 70148 USA \\ $*^{3}$ Department of Engineering Math. \& Physics, University of Alexandria, Alexandria 21544 Egypt
}

\begin{abstract}
Deformation due to pressure variations of human blood vessel embedded in a soft tissue is considered. Due to symmetry it suffices to consider only a cross section subject to a uniform pressure acting normally through the vessel's wall. The nonlinearity of the vessel's wall is modeled by an isotropic hyperelastic Fung type stress strain model. The surrounding soft tissue is modeled by infinitely many nonlinear springs with two parameters. The equations of equilibrium are formulated as nonlinear boundary value problem. A differential correction numerical scheme is used to solve the nonlinear governing equations and the symmetrical post-buckling shapes of the cross section are presented.
\end{abstract}

Keywords: Blood vessel, Blood pressure, Buckling and Post-buckling, Nonlinear boundary value problem

\section{Introduction}

Buckling and post-buckling of an embedded collapsible blood vessel affects the blood flow rate through this vessel. The vessel may collapse due to an increase in the pressure difference between the inside and outside of the vessel. The surrounding soft tissue is modelled as infinitely many number of linear [1] or nonlinear [2] springs. As the pressure difference exceeds a critical value, buckling occurs leading to symmetrical shapes of the vessel's wall with $N \geq 2$ axes of symmetry. Increasing the pressure further gives post-buckling shapes. Both, buckling and postbuckling shapes are affected by the characteristics of the soft tissue and the vessel's wall parameters. The phenomena of collapsible tubes was used to help explaining blood flow rate and blood pressure in veins $[3,4]$. The relation between the angular displacement of a local tangent to the vessel with an arbitrary axis and the arch length describes the shape of the cross section. In the linearized problem this relation is linear giving a circular cross section. The jacobian matrix of the system evaluated at this special solution becomes singular for certain values of the pressure difference, the smallest of which is called the critical buckling load. Beyond this value of the pressure difference, periodic shapes of the cross section are obtained. The periodicity conditions of such shapes will be used in solving the governing equations, numerically.
In the second section the governing equations will be formulated. In the third section an iterative corrective numerical technique will be used to solve the nonlinear governing equations. In the fourth section graphical representations of the post-buckling shapes of the cross section are presented and the effect of nonlinearities are discussed. Finally, some conclusions are drawn in the fifth section.

\section{Governing Equations}

\subsection{Stress Strain Models}

The stress strain model provided by $[5,6]$, based on bending experiment, is used to model the blood vessel wall

$\sigma=E \varepsilon+\beta\left(e^{\alpha \varepsilon}-1-\alpha \varepsilon\right)$,

where $\sigma$ is the stress, $E$ is the Young's modulus, $\varepsilon$ is the strain, and $\alpha, \beta$ are material parameters. For small values of $\alpha \varepsilon$, (1) simplifies to

$$
\sigma=E \varepsilon+\beta \alpha^{2}|\varepsilon| \varepsilon
$$

The surrounding tissue is modeled as nonlinear springs obeying the generalized Hooke's law

*Corresponding author: Engineering Building, Science Department Texas A\&M University-Qatar, 23874, Doha Qatar, E-mail: mohammed.ghazy@qatar.tamu.edu, Phone: +97470475375 Fax: +97444230011 
$F(s)=k_{1} s+k_{2} g(s)$

where $k_{1}, k_{2}$ are positive constants and $g(s)$ is an odd function of $s$.

\subsection{Equilibrium Equations}

Figure 1 shows an elemental length $d s$ of the blood vessel's cross section in which $\theta$ is the angular displacement measured from the $x$ axis, $T$ is the tensional force, $M$ is the bending moment, $S$ is the shearing force, and $q_{n}^{\prime}, q_{t}^{\prime}$ are stresses along the local intrinsic coordinates

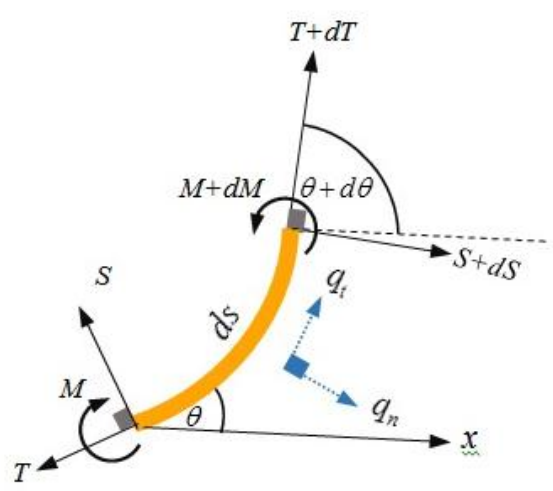

Figure 1. An Element Length

Force equilibrium along the normal and tangential directions respectively gives

$T d \theta=q_{n}^{\prime} d s^{\prime}+d S$

$q_{t}^{\prime} d s^{\prime}+S d \theta+d T=0$

Equilibrium of moments gives

$d M=S d s^{\prime}$

Equations (4)-(6) have four unknown quantities, functions of the independent variable $s^{\prime}$, and one additional equation is needed. This equation is

$$
M=\int_{A} \sigma \delta d A
$$

where $\delta$ is the strain to curvature ratio and $A$ is the cross-sectional area of the element. Substituting (2) into (7) gives

$$
M=\int_{A}\left(E z \frac{d \theta}{d s^{\prime}}+\beta \alpha^{2}\left|z \frac{d \theta}{d s^{\prime}}\right| z \frac{d \theta}{d s^{\prime}}\right) z d A
$$

or

$$
M=E I_{2} \frac{d \theta}{d s^{\prime}}+\beta \alpha^{2} I_{3}\left|\frac{d \theta}{d s^{\prime}}\right| \frac{d \theta}{d s^{\prime}}
$$

where $I_{2}$ and $I_{3}$ are the second and third area moment of inertia respectively. Substituting (9) into (6) the shear force can be represented as

$$
S=E I_{2} \frac{d^{2} \theta}{d s^{\prime 2}}+2 \beta \alpha^{2} I_{3}\left|\frac{d \theta}{d s^{\prime}}\right| \frac{d^{2} \theta}{d s^{\prime 2}}
$$

Substituting (10) into (5) yields

$q_{t}^{\prime}+\left(E I_{2} \frac{d^{2} \theta}{d s^{\prime 2}}+2 \beta \alpha^{2} I_{3}\left|\frac{d \theta}{d s^{\prime}}\right| \frac{d^{2} \theta}{d s^{\prime 2}}\right) \frac{d \theta}{d s^{\prime}}+\frac{d T}{d s^{\prime}}=0^{(11)}$

Dividing (4) by $d s^{\prime}$ gives

$T \frac{d \theta}{d s^{\prime}}-\frac{d S}{d s^{\prime}}=q_{n}^{\prime}$

Differentiating (12) with respect to $s^{\prime}$ gives

$$
\frac{d T}{d s^{\prime}} \frac{d \theta}{d s^{\prime}}+T \frac{d^{2} \theta}{d s^{\prime 2}}-\frac{d^{2} S}{d s^{\prime 2}}=\frac{d q_{n}^{\prime}}{d s^{\prime}}
$$

We introduce dimensionless quantities normalized by system parameters defined by:

$s=\frac{s^{\prime}}{R}, q_{n}=\frac{q_{n}^{\prime} R^{3}}{E I_{2}}, q_{t}=\frac{q_{t}^{\prime} R^{3}}{E I_{2}}, \hat{\beta}=\frac{2 \beta \alpha^{2} I_{3}}{E I_{2} R}$

Differentiating (10) with respect to $s^{\prime}$ and using (14) gives

$$
\frac{d S}{d s}=\left(1+\hat{\beta}\left|\frac{d \theta}{d s}\right|\right) \frac{d^{3} \theta}{d s^{3}}+\hat{\beta} \operatorname{sign}\left(\frac{d \theta}{d s}\right)\left(\frac{d^{2} \theta}{d s^{2}}\right)^{2}
$$

Substituting (15) into (12) and using (14) gives

$$
T \frac{d \theta}{d s}=\left(1+\hat{\beta}\left|\frac{d \theta}{d s}\right|\right) \frac{d^{3} \theta}{d s^{3}}+\hat{\beta} \operatorname{sign}\left(\frac{d \theta}{d s}\right)\left(\frac{d^{2} \theta}{d s^{2}}\right)^{2}+q_{n}
$$

From (5) and (14) we get

$$
\frac{d T}{d s}=-\left(1+\hat{\beta}\left|\frac{d \theta}{d s}\right|\right) \frac{d \theta}{d s} \frac{d^{2} \theta}{d s^{2}}-q_{t}
$$


Figure 2 depicts the geometry of the deflection of element length allowing point $\mathbf{A}$ to move to an arbitrary location $\mathbf{B}$ with coordinates $(x, y)$. We obtain the equations

$\frac{d x}{d s}=\cos \theta, \quad \frac{d y}{d s}=\sin \theta$

and

$$
d=\sqrt{(a \cos s-x)^{2}+(a \sin s-y)^{2}}
$$

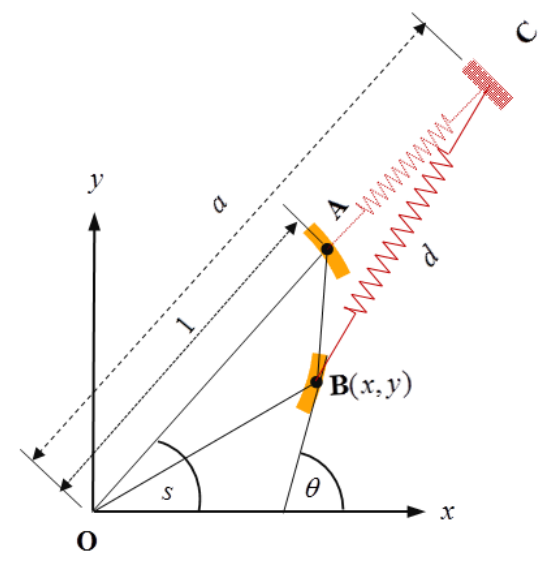

Figure 2. Deflection Geometry

Since the elongation in the spring due to deflection is given by

$$
z=\overline{\mathbf{B C}}-\overline{\mathbf{A C}}=d-a+1
$$

the spring force per unit area per unit length is given by

$$
F(z)=k_{1} z+k_{2} g(z)
$$

This spring force in (21) together with the pressure difference

$$
P=P_{e}-P_{i}-F_{0},
$$

where $F_{0}$ is initial spring force, give the stresses in the normal and tangential directions

$$
\begin{aligned}
& q_{t}=\frac{F(z)}{d}[\cos \theta(a \cos s-x)+\sin \theta(a \sin s-y)] \\
& q_{n}=\frac{F(z)}{d}[\sin \theta(a \cos s-x)-\cos \theta(a \sin s-y)]-P
\end{aligned}
$$

Knowing that the cross section may take symmetrical shapes with $N \geq 2$ axes of symmetry. The boundary condition for such symmetrical shapes are

$$
\begin{gathered}
\left.\theta(0)=\frac{\pi}{2},\left.\frac{d^{2} \theta}{d s^{2}}\right|_{s=0}=0, y 0\right)=0 \\
\theta\left(\frac{2 \pi}{N}\right)=\frac{\pi}{2}+\frac{2 \pi}{N},\left.\frac{d^{2} \theta}{d s^{2}}\right|_{s=\frac{2 \pi}{N}}=0,\left.\left(x^{2}+y^{2}\right)\right|_{s=0}=\left.\left(x^{2}+y^{2}\right)\right|_{s=\frac{2 \pi}{N}}
\end{gathered}
$$

\section{Numerical Solution}

The equilibrium equations can be written as a vector differential equation

$$
\frac{d \mathbf{q}}{d s}=\mathbf{f}(\mathbf{q}, s)
$$

where $\mathbf{q}$ is the state vector and $\mathbf{f}(\mathbf{q}, s)$ is a nonlinear vector function

$$
\mathbf{q}=\left\{\begin{array}{c}
\theta \\
\frac{d \theta}{d s} \\
\frac{d^{2} \theta}{d s^{2}} \\
T \\
x \\
y
\end{array}\right\}, \mathbf{f}=\left\{\begin{array}{c}
q_{2} \\
q_{3} \\
\frac{\left(q_{2} q_{4}-q_{n}-\hat{\beta} \operatorname{sign}\left(q_{2}\right) q_{3}^{2}\right)}{\left(1+\hat{\beta}\left|q_{2}\right|\right)} \\
-\left(1+\hat{\beta}\left|q_{2}\right|\right) q_{2} q_{3}-q_{t} \\
\cos q_{1} \\
\sin q_{1}
\end{array}\right\}
$$

with the boundary conditions

$$
\begin{aligned}
& q_{1}(0)=\frac{\pi}{2}, q_{3}(0)=0, q_{6}(0)=0 \\
& q_{1}\left(\frac{2 \pi}{N}\right)=\frac{\pi}{2}+\frac{2 \pi}{N}, q_{3}\left(\frac{2 \pi}{N}\right)=0 \\
& \left.\left(q_{5}^{2}+q_{6}^{2}\right)\right|_{s=0}=\left.\left(q_{5}^{2}+y_{6}^{2}\right)\right|_{s=\frac{2 \pi}{N}}
\end{aligned}
$$

The problem (26), (28) is a nonlinear boundary value problem which we solve numerically using a differential correction scheme as an application of Newton's method [7]. In such a scheme the state equations, together with the associated state transition matrix differential equation, are numerically integrated using arbitrary initial conditions for $s=[0,2 \pi / N]$. If, at $s=2 \pi / N$, the periodicity conditions are satisfied the correction process is terminated. Otherwise the Newton's equation is applied to update the initial conditions. 


\section{Numerical Results}

Figures 3-6 shows post-buckling shapes for a blood vessel in which $k_{1}=3, k_{2}=1, g(z)=\tanh (z)$ and $\hat{\beta}=0.05$.

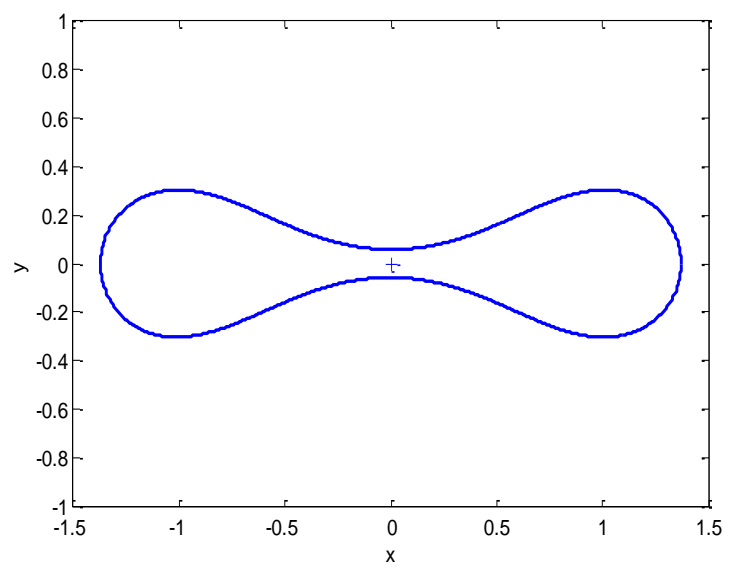

Figure 3. $N=2, P=7.7$

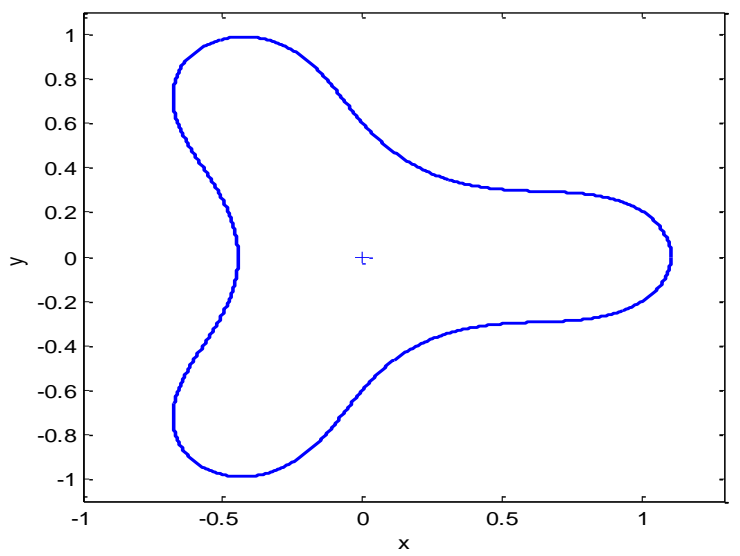

Figure 4. $N=3, P=14$

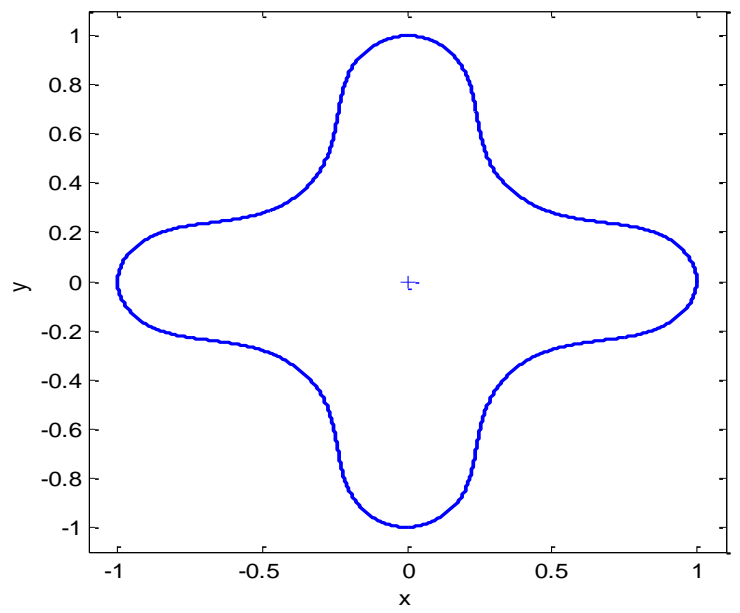

Figure 5. $N=4, P=26.2$

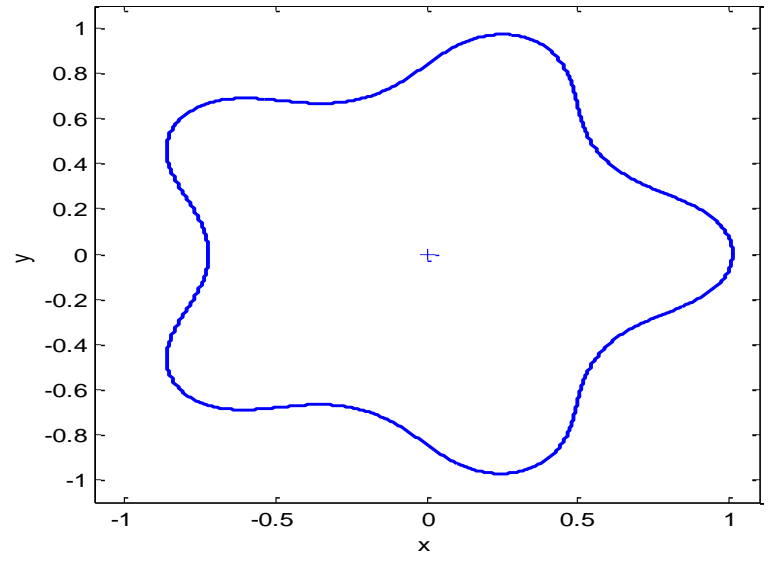

Figure 6. $N=5, P=33$

Figures 7-9 show the effect of the nonlinearity represented by $\hat{\beta}$ on the initial conditions $\frac{d \theta}{d s}(0), T(0), x(0)$ required for convergence for the case at $N=4, P=26.2, k_{1}=3, k_{2}=1$, and $g(z)=\tanh (z)$. Both $\frac{d \theta}{d s}(0), T(0)$ decrease when $\hat{\beta}$ increases while $x(0)$ increases by increasing $\hat{\beta}$

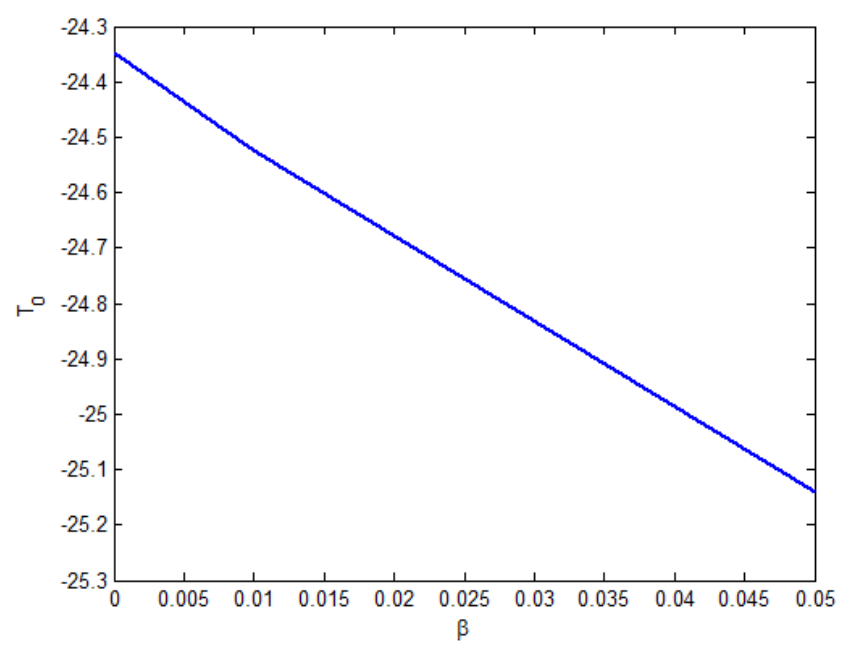

Figure 7. $\hat{\beta}$ vs $\frac{d \theta}{d s}(0)$ 


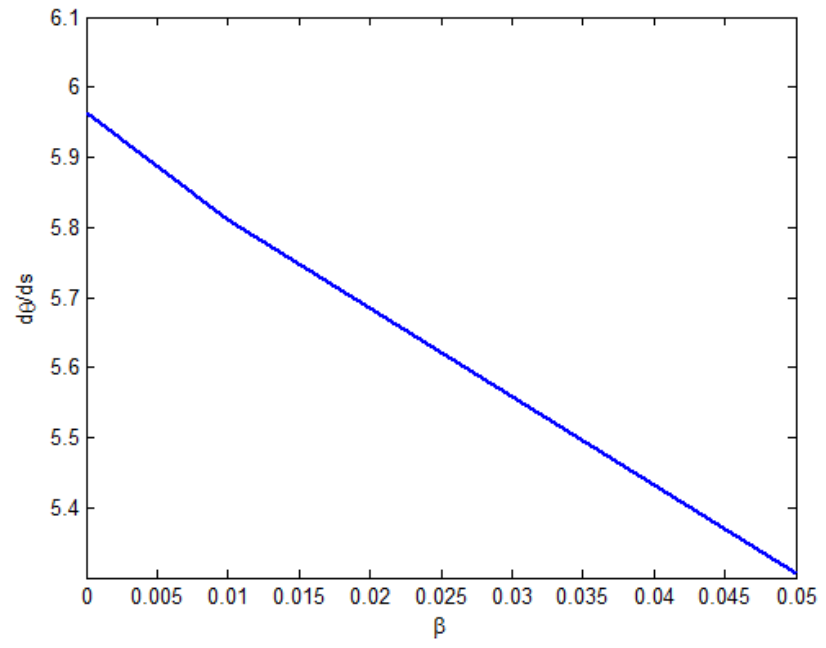

Figure 8. $\hat{\beta}$ vs $T(0)$

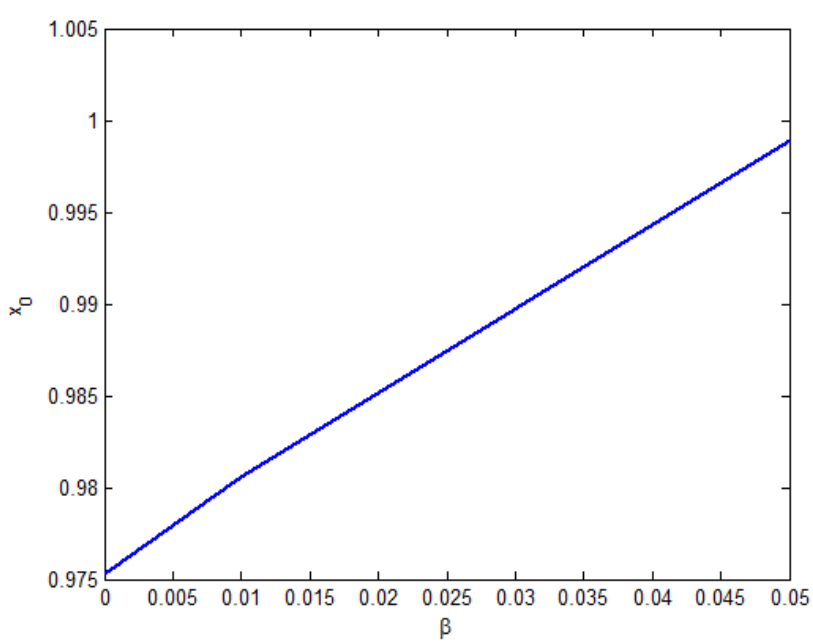

Figure 9. $\hat{\beta}$ vs $x(0)$

\section{Conclusions}

Equations of equilibrium that govern the deformation of a blood vessel embedded in soft tissue are obtained using a nonlinear stress-strain model presented by Fung $[5,6]$. The equations are integrated numerically and effects of the model's nonlinearity on the post buckling shapes are demonstrated.

\section{Acknowledgements}

This research is partially supported by the research office at Texas A\&M University in Qatar.

\section{References}

[1] Wang, C.Y., Watson, L.T., and Kamat, M.P., Buckling, Postbuckling, and Flow Rate Through a Tethered Elastic Cylinder Under External Pressure, J Appl. Mech., 1983;50:13-18.

[2] Cypher A., Elgindi, M., Kouriachi, H., Peschman, D., and Shotwell, R., Flow Rate Through a Blood Vessel Deformed Due to a Uniform Pressure, Journal of Biomaterial and Nanobiotechnology,2011;2:369377.

[3] Pedley, T. J., Brook, B. S., Seymour, R. S., Blood Pressure and Flow Rate in the Giraffe Jugular Vein, Phil. Trans: R. Soc. Lond. B., 1996;351:855-66.

[4] Fung, Y.C., Biomechanics, Mechanical Properties of Living Tissues, Springer-Berlag, 1990.

[5] Tanaka, T. T., and Fung, Y. C, "Elastic and Inelastic Properties of the Canine Aorta and Their Variation along the Aortic Tree," J. Biomech., 1974;7:357-70.

[6] Fung, Y.C.,S. Q. Liu, and J. B. Zhou Remodeling of the Constitutive Equation While a Blood Vessel Remodels Itself Under Stress, Journal of Biomechanical Engineering, 1993;115:453-59.

[7] Ghazy, M., and Newman. Solvability Conditions at Equilibrium Points. AIAA/AAS Astrodynamics Specialist Conference, Toronto, Ontario, Canada, 2-5 August 2010. 